\title{
Fourier Series Approximation for Max Operation in Non-Gaussian and Quadratic Statistical Static Timing Analysis
}

\author{
Lerong Cheng, Member, IEEE, Fang Gong, Student Member, IEEE, Wenyao Xu, Student Member, IEEE, \\ Jinjun Xiong, Member, IEEE, Lei He, Senior Member, IEEE, and Majid Sarrafzadeh, Fellow, IEEE
}

\begin{abstract}
The most challenging problem in the current block-based statistical static timing analysis (SSTA) is how to handle the max operation efficiently and accurately. Existing SSTA techniques suffer from limited modeling capability by using a linear delay model with Gaussian distribution, or have scalability problems due to expensive operations involved to handle non-Gaussian variation sources or nonlinear delays. To overcome these limitations, we propose efficient algorithms to handle the max operation in SSTA with both quadratic delay dependency and non-Gaussian variation sources simultaneously. Based on such algorithms, we develop an SSTA flow with quadratic delay model and non-Gaussian variation sources. All the atomic operations, max and add, are calculated efficiently via either closed-form formulas or low dimension (at most 2-D) lookup tables. We prove that the complexity of our algorithm is linear in both variation sources and circuit sizes, hence our algorithm scales well for large designs. Compared to Monte Carlo simulation for non-Gaussian variation sources and nonlinear delay models, our approach predicts the mean, standard deviation and $95 \%$ percentile point with less than $2 \%$ error, and the skewness with less than $10 \%$ error.
\end{abstract}

Index Terms - Process variation, statistical static timing analysis (SSTA), timing.

\section{INTRODUCTION}

A $\mathrm{S}$ continuous CMOS technology scaling, process variation has become a potential show-stopper if not appropriately handled [1]-[6]. Statistical static timing analysis (SSTA) has thus become the frontier research topic in recent years in combating such variation effects. There are two types of SSTA methods, path-based SSTA [7]-[10] and block-based SSTA [11]-[19]. The goal of SSTA is to parameterize timing characteristics of the timing graph as a function of the underlying sources of process parameters that are modeled as

Manuscript received November 09, 2010; revised April 19, 2011; accepted May 05, 2011. Date of publication June 30, 2011; date of current version June 14, 2012.

L. Cheng is with SanDisk Corporation, Milpitas, CA 95035 USA (e-mail: lerong@ee.ucla.edu).

F. Gong, W. Xu, and L. He are with the Department of Electrical Engineering, University of California, Los Angeles, CA 90095 USA (e-mail: fang08@ee. ucla.edu; wxu@ee.ucla.edu; 1he@ee.ucla.edu).

J. Xiong is with IBM Thomas J. Watson Research Center, Yorktown Heights, NY 10598 USA (e-mail: jinjun@ee.ucla.edu).

M. Sarrafzadeh is with the Department of Computer Science, University of California, Los Angeles, CA 90095 USA (e-mail: majid@cs.ucla.edu).

Color versions of one or more of the figures in this paper are available online at http://ieeexplore.ieee.org.

Digital Object Identifier 10.1109/TVLSI.2011.2157843 random variables. By performing SSTA, designers can obtain the timing distribution (yield) and its sensitivity to various process parameters. Such information is of tremendous value for both timing sign-off and design optimization for robustness and high profit margins.

The block-based SSTA is the most efficient SSTA method in recent years. In block-based SSTA, there are two major atomic operations, max and add. The add operation is simple, however, the max operation is much more complex. How to perform the max operation is the hardest problem in block-based SSTA. Although many studies have been done on this in recent years, the problem is far from being solved completely. For example, [11] and [12] assumed that all variation sources are Gaussian and independent of each other. Based on a linear delay model, [12] proposed a linear-time algorithm for SSTA, in which both atomic operations, max and add, can be performed efficiently via the concept of tightness probability. Because all variation sources are assumed to be Gaussian, so is the delay distribution under the linear delay model.

Such a Gaussian assumption is, however, no longer tolerable as more complicated or large-scale variation sources are taken into account in the nanometer manufacturing regime. For example, via resistance is known to be non-Gaussian with asymmetric distribution [17]; and dopant concentration is more suitably modeled as a Poisson distribution [16]. In addition, the linear dependency of delay on the variation sources is also not accurate, especially when variation becomes large [20]. For example, gate delay is inherently a nonlinear function of channel length and Vth [13], [17], which are two common sources of variation. Similarly, interconnect delay is also a nonlinear function of interconnect geometries [13], [14], which vary because of chemical-mechanical polishing. These combined non-Gaussian and nonlinear variation effects invalidate the linear delay model with Gaussian assumption in the existing SSTA.

Recently, non-Gaussian variation sources were addressed in [16], where independent component analysis (ICA) was used to find a set of independent components (not necessary Gaussian) to approximate the correlated non-Gaussian random variables. To do this, however, a complicated moment matching algorithm has to be used to make those atomic statistical operations feasible. Reference [21] introduced a conditional linear approximation of the max operation and captured all types of correlation. However, both of the above works are still based on a linear delay model, which cannot capture the nonlinear dependency of 
delays on process parameters. To capture these nonlinear dependency effects, [13] and [14] proposed to use a quadratic delay model for SSTA. But to contain the complexity, they had to assume that all variation sources must follow a Gaussian distribution, even though the delay $D$ itself may not be Gaussian. To compute $\max \left(D_{1}, D_{2}\right)$, [13] first developed closed form formulas to compute the mean and variance of the quadratic form. It then treats $D_{1}$ and $D_{2}$ as a Gaussian distribution to obtain the tightness probability. There is, however, no justification on why the tightness probability formula developed for Gaussian distributions can be applied for non-Gaussian distributions. [14] tried to re-construct $\max \left(D_{1}, D_{2}\right)$ through moment matching. To obtain those moments, however, many expensive numerical integration (2-D) operations have to be applied.

There are some existing studies [15], [17]-[19] trying to handle both nonlinear and non-Gaussian effects simultaneously. However, [15] computes $\max \left(D_{1}, D_{2}\right)$ by regression based on Monte Carlo simulation, which obviously is slow; [17] computed the max operation through tightness probability while [19] applied moment matching to reconstruct the max. However, to do so, both had to resort to expensive multi-dimension numerical integration techniques. [17] requires multi-dimension numerical integration to calculate the tightness probability and the number of integration dimensions depends on the number of non-Gaussian variation sources. [19] applies two-dimensional numerical integration to obtain the joint moments between variation sources and max. It is more efficient than [15] and [17]. But 2-D numerical integration is still an expensive operation for block-based SSTA. Hence scalability of such methods to handle a large number of non-Gaussian variation sources is limited. [18] handled the atomic operations by approximating the gate delay using a set of orthogonal polynomials, which needs to be constructed for different variation distributions.

In this work, we propose a novel method to handle the max operation for quadratic delay model with non-Gaussian variation sources. Based on such model, we develop a nonlinear and non-Gaussian SSTA technique ( $n^{2}$ SSTA). The major advantages of this technique are multi-fold: 1) both nonlinear dependency and non-Gaussian variation sources are handled simultaneously for timing analysis; 2) all statistical atomic operations, max and add, are performed efficiently via either closed-form formulas or low dimension (at most 2-D) lookup tables; 3 ) the complexity of the $n^{2}$ SSTA algorithm is linear in both number of variation sources and circuit sizes. Compared to Monte Carlo simulation for non-Gaussian variation sources and nonlinear delay models, our approach predicts the mean, standard deviation and $95 \%$ percentile point with less than $2 \%$ error, and the skewness with less than $10 \%$ error.

The rest of the paper is organized as follows. Section II presents our nonlinear and non-Gaussian delay modeling and the basic block-based SSTA flow. Section III discuss our algorithm of the max operation with quadratic delay model and non-Gaussian variation sources. Section IV simplifies the method to handle linear delay model. We present experiments in Section V, and concludes this paper in Section VI.

\section{Preliminaries AND Modeling}

\section{A. Quadratic Delay Modeling}

In general, device or interconnect delays of a design are a complicated nonlinear function of the underlying process parameters and it can be described as

$$
D=F\left(X_{1}, X_{2}, \ldots, X_{i}, \ldots\right)
$$

where the process parameters (such as channel length and threshold voltage) are modeled as a random variable $X_{i}$. In reality, the exact form of function $F$ is not known, and $X_{i}$ are not necessarily Gaussian.

The simplest approximation is the first- and second-order Taylor expansion as shown as follows:

$$
\begin{aligned}
D & \approx d_{0}+\sum a_{i} X_{i} \\
D & \approx d_{0}+\sum a_{i} X_{i}+\sum b_{i} X_{i}^{2}+\sum_{i \neq k} b_{i, k} X_{i} X_{k}
\end{aligned}
$$

where $d_{0}$ is the nominal value of $D ; a_{i}$ and $b_{i}$ are the first- and second-order sensitivities of $D$ to $X_{i}$, respectively; and $b_{i, k}$ are the sensitivity to the joint variation of $X_{i}$ and $X_{k}$. (2) is called the first-order canonical form, and is widely used for SSTA [11], [12]; whereas (3) is called the quadratic delay model, and has been studied in [13]-[15], [20]. But Gaussian assumptions limit their modeling capability and prevent them from reflecting the reality.

Therefore, we propose a different quadratic model as follows:

$$
D=d_{0}+\sum\left(a_{i} X_{i}+b_{i} X_{i}^{2}\right)+r R
$$

where $X_{i}$ represents global sources of variation, and $R$ represents purely independent random variation which is modeled as a Gaussian random variable. Unlike previous work, we allow $X_{i}$ to follow arbitrary random distributions. We refer to the delay model (4) as general canonical form in this paper.

\section{B. Block-Based SSTA Framework}

To compute the arrival time and required arrival time in a block-based SSTA framework, four atomic operations are sufficient, i.e., addition, subtraction, maximum, and minimum. Because of the symmetry between addition and subtraction (similarly maximum and minimum) operations, in the following, we will only discuss operations on addition and maximum. Given $D_{1}$ and $D_{2}$ in the form of (4)

$$
\begin{aligned}
& D_{1}=d_{10}+\sum\left(a_{1 i} X_{i}+b_{1 i} X_{i}^{2}\right)+r_{1} R_{1} \\
& D_{2}=d_{20}+\sum\left(a_{2 i} X_{i}+b_{2 i} X_{i}^{2}\right)+r_{2} R_{2}
\end{aligned}
$$

we want to compute the $D_{s}=D_{1}+D_{2}$ and $D_{m}=$ $\max \left(D_{1}, D_{2}\right)$ in the following form:

$$
\begin{gathered}
D_{s}=d_{s 0}+\sum\left(a_{s i} X_{i}+b_{s i} X_{i}^{2}\right)+r_{s} R_{s} \\
D_{m}=d_{m 0}+\sum\left(a_{m i} X_{i}+b_{m i} X_{i}^{2}\right)+r_{m} R_{m} .
\end{gathered}
$$


The add operation is straight forward. The coefficients of $D_{s}$ can be computed by adding the correspondent coefficients of $D_{1}$ and $D_{2}$

$$
\begin{aligned}
& d_{s 0}=d_{01}+d_{02} ; \quad a_{s i}=a_{1 i}+a_{2 i} \\
& b_{s i}=b_{1 i}+b_{2 i} ; \quad r_{s}=\sqrt{r_{1}^{2}+r_{2}^{2}} .
\end{aligned}
$$

The max operation is much complexer than the add operation, and we will discussed this in detail in Section III.

\section{MAX For Quadratic Delay Model}

The max operation is the hardest operation for block-based SSTA. In this section, we propose a novel technique to efficiently compute the max of two general canonical forms, i.e., $D_{m}=\max \left(D_{1}, D_{2}\right)$. Without loss of the generality, in the following of this paper, we assume that $E\left[D_{1}\right]>E\left[D_{2}\right]$. We first compute the mean and variance of $V=D_{1}-D_{2}$, if $\mu_{V}>3 \sigma_{V}$, then $D_{m}=D_{1}$. Otherwise, we compute the skewness of $V$ and $E[\max (V, 0)]$. After that, we approximate the joint PDF of $V$ and $X_{i}$ 's with Fourier Series and compute the joint moments between $V$ and $X_{i}$ 's. Finally, we compute the joint moments between $D_{m}$ and $X_{i}$ 's and reconstruct the canonical form of $D_{m}$. In the following, we discuss our approach in details.

\section{A. Moments of $V$}

From the definition of $V$ as discussed in the previous section, the max operation can be rewritten as

$\max \left(D_{1}, D_{2}\right)=\max \left(D_{1}-D_{2}, 0\right)+D_{2}=\max (V, 0)+D_{2}$.

According to (5) and (6), $V$ can be written as the following form:

$$
V=d_{0}+\sum\left(a_{i} X_{i}+b_{i} X_{i}^{2}\right)+r_{1} R_{1}-r_{2} R_{2}
$$

where $a_{i}=a_{1 i}-a_{2 i}, b_{i}=b_{1 i}-b_{2 i}$, and $d_{0}=d_{01}-d_{02}$. In order to compute the central moments of $V$, we first rewrite $V$ to the following form:

$$
V=d_{0}^{\prime}+\sum_{i=1}^{n} Z_{i}+r_{1} R_{1}-r_{2} R_{2}
$$

where

$$
\begin{aligned}
& d_{0}^{\prime}=d_{0}+\sum_{i=1}^{n} b_{i} \\
& Z_{i}=a_{i} X_{i}+b_{i} X_{i}^{2}-b_{i}
\end{aligned}
$$

From the above equations, it is easy to compute the first three moments of $Z_{i}$ 's

$$
\begin{aligned}
E\left[Z_{i}\right]= & 0 \\
E\left[Z_{i}^{2}\right]= & b_{i}^{2}\left(m_{i}(4)-1\right)+2 a_{i} b_{i} m_{i}(3)+a_{i}^{2} \\
E\left[Z_{i}^{3}\right]= & 3 a_{i} b_{i}^{2}\left(m_{i}(5)-2 m_{i}(3)\right)+3 a_{i}^{2} b_{i}\left(m_{i}(4)-1\right) \\
& +a_{i}^{3} m_{i}(3)+b_{i}^{3}\left(m_{i}(6)-3 m_{i}(4)+2\right)
\end{aligned}
$$

Because $X_{i}$ 's are mutually independent random variables, $Z_{i}$ 's are independent random variables with zero mean. Therefore, the first three central moments of $V$ are

$$
\begin{aligned}
\mu_{V} & =E[V]=d_{0}^{\prime} \\
\sigma_{V}^{2} & =E\left[\left(V-\mu_{V}\right)^{2}\right]=r_{1}^{2}+r_{2}^{2}+\sum_{i=1}^{n} E\left[Z_{i}^{2}\right] \\
m_{V}(3) & =E\left[\left(V-\mu_{V}\right)^{3}\right]=r_{1}^{3}+r_{2}^{3}+\sum_{i=1}^{n} E\left[Z_{i}^{3}\right] .
\end{aligned}
$$

With the central moments, the skewness of $V$ can be computed as

$$
\gamma_{V}=m_{V}(3) / \sigma_{V}^{3} .
$$

After computing the mean and variation of $V$, we can quickly compute some trivial max operation. When $\mu_{V}$ is larger than the $m-\sigma$ value, that is, $\mu_{V}>m \cdot \sigma_{V}$, which means the probability that $D_{1}>D_{2}$ is very high, we may let $\max \left(D_{1}, D_{2}\right) \approx D_{1}$. The range value $m$ can be set by the users, in the following of this paper, we let $m=3$.

\section{B. Mean of the Max Operation}

In order to reconstruct the canonical form of $D_{m}$, we first need to compute $\mu_{V m}=E[\max (V, 0)]$. When $V$ is a nonGaussian random variable, exact computation of $\mu_{V m}$ is difficult in general. Therefore, we propose to use the following two-step procedure to approximately compute $\mu_{V m}$. In the first step, we approximate the non-Gaussian random variable $V$ as a quadratic function of a standard Gaussian random variable $W$ similar to [18] and [24], i.e.,

$$
V \approx P(W)=c_{2} \cdot W^{2}+c_{1} \cdot W+c_{0} .
$$

The coefficients $c_{2}, c_{1}$, and $c_{0}$ can be obtained by matching $P(W)$ and $V$ 's mean, variance, and skewness simultaneously, i.e.,

$$
\begin{aligned}
\mu_{V} & =E\left[c_{2} \cdot W^{2}+c_{1} \cdot W+c_{0}\right]=c_{2}+c_{0} \\
\sigma_{V}^{2} & =E\left[\left(c_{2} \cdot W^{2}+c_{1} \cdot W+c_{0}-\mu_{V}\right)^{2}\right]=2 c_{2}^{2}+c_{1}^{2} \\
\gamma_{V} \cdot \sigma_{V}^{3} & =E\left[\left(c_{2} \cdot W^{2}+c_{1} \cdot W+c_{0}-\mu_{V}\right)^{3}\right]=8 c_{2}^{3}+6 c_{2} c_{1}^{2} .
\end{aligned}
$$

As shown in [24], solving the above equations, $c_{2}$ will be one of the following values:

$$
\begin{aligned}
& c_{2,1}=-\frac{2 \sigma_{V}^{2}+\Delta^{2 / 3}}{2 \Delta^{1 / 3}} \\
& c_{2,2}=\frac{2(1+j \sqrt{3}) \sigma_{V}^{2}+(1-j \sqrt{3}) \Delta^{2 / 3}}{4 \Delta^{1 / 3}} \\
& c_{2,3}=\frac{2(1-j \sqrt{3}) \sigma_{V}^{2}+(1+j \sqrt{3}) \Delta^{2 / 3}}{4 \Delta^{1 / 3}}
\end{aligned}
$$

where $\Delta=\gamma_{V} \cdot \sigma_{V}^{3}+j \sqrt{8 \sigma_{V}^{6}-\gamma_{V}^{2} \cdot \sigma_{V}^{6}}$, with $j=\sqrt{-1}$. The mean, variance, and skewness of $V$ can be computed from (19), (20), and (22), respectively. It is proved in [24] that, when $\left|\gamma_{V}\right|<2 \sqrt{2}$ there exists one and only one of the above three 
values in the range $\left|c_{2}\right|<\sigma_{V} / \sqrt{2}$. We will pick such value for $c_{2}$. When $\left|\gamma_{V}\right|>2 \sqrt{2}$, we may let

$$
c_{2}= \begin{cases}\sigma_{V} / \sqrt{2} & \gamma_{V}>2 \sqrt{2} \\ -\sigma_{V} / \sqrt{2} & \gamma_{V}<-2 \sqrt{2}\end{cases}
$$

It is proved in [18] that the above equations gives $P(W)$ which matches the mean and variance of $V$ and has the skewness closest to $\gamma_{V}$. With $c_{2}, c_{1}$, and $c_{0}$ can be computed as

$$
\begin{aligned}
& c_{1}=\sqrt{\sigma_{V}^{2}-2 c_{2}^{2}} \\
& c_{0}=\mu_{V}-c_{2} .
\end{aligned}
$$

After obtaining the coefficients $c_{2}, c_{1}$, and $c_{0}$ in the second step, we approximate the exact mean of $\max (V, 0)$ by the exact mean of $\max (P(W), 0)$, i.e.,

$$
\mu_{V} m \approx E[\max (P(W), 0)]=\int_{P(w)>0} P(w) \phi(w) d w
$$

where $\phi(\cdot)$ is the PDF of the standard normal distribution. In the above equation, the integration range $P(w)>0$ can be computed in the following four different cases.

Case 1) $c_{2}>0$

$$
P(w)>0 \Leftrightarrow w<t_{1} \vee w>t_{2}
$$

where

$$
\begin{aligned}
& t_{1}=\left(-c_{1}-\sqrt{c_{1}^{2}-4 c_{2} c_{0}}\right) / 2 c_{2} \\
& t_{2}=\left(-c_{1}+\sqrt{c_{1}^{2}-4 c_{2} c_{0}}\right) / 2 c_{2} .
\end{aligned}
$$

Case 2) $c_{2}<0$

$$
P(w)>0 \Leftrightarrow t_{2}<w<t_{1} .
$$

Case 3) $c_{2}=0 \wedge c_{1}>0$

$$
P(w)>0 \Leftrightarrow w>-c_{0} / c_{1} .
$$

Case 4) $c_{2}=0 \wedge c_{1}<0$

$$
P(w)>0 \Leftrightarrow w<-c_{0} / c_{1} .
$$

Knowing the integration range, we can compute $E[\max (P(W), 0)]$ under such four cases

$$
\begin{aligned}
& E[\max (P(W), 0)] \\
& \quad= \begin{cases}\left(c_{2}+c_{0}\right)\left(1+\Phi\left(t_{1}\right)-\Phi\left(t_{2}\right)\right) & \\
\quad+\left(c_{1}+t_{1}\right)\left(\phi\left(t_{2}\right)-\phi\left(t_{1}\right)\right) & c_{2}>0 \\
\left(c_{2}+c_{0}\right)\left(\Phi\left(t_{1}\right)-\Phi\left(t_{2}\right)\right) & \\
\quad+\left(c_{1}+t_{1}\right)\left(\phi\left(t_{2}\right)-\phi\left(t_{1}\right)\right) & c_{2}<0 \\
c_{0} \cdot \Phi\left(c_{0} / c_{1}\right)+c_{1} \cdot \phi\left(c_{0} / c_{1}\right) & c_{2}=0 \wedge c_{1}>0 \\
c_{0} \cdot\left(1-\Phi\left(c_{0} / c_{1}\right)\right)-c_{1} \cdot \phi\left(c_{0} / c_{1}\right) & c_{2}=0 \wedge c_{1}<0\end{cases}
\end{aligned}
$$

where $\Phi(\cdot)$ is the cumulative density function (CDF) of the standard normal distribution. According to (41), we can compute $\mu_{V m}$ easily through analytical formulas.

\section{Joint Moments Between $\max (V, 0)$ and Variation Sources}

In the previous section, we compute the mean of $\max (V, 0)$. In order to reconstruct the canonical form of $D_{m}$, we also need to know the joint moments between $V$ and the variation sources $X_{i}$ 's. Because $V$ and $X_{i}$ 's are correlated non-Gaussian random variables, the computation of the joint moments between them is complex. In this section, we will introduce a new method based on Fourier Series expansion to solve such problem.

In order to compute the joint moments, we first approximate the joint PDF of $V$ and the $i$ th variation sources $X_{i}, f_{i}\left(v, x_{i}\right)$, by its first $K$ th order 2-D Fourier Series within the $3 \sigma$ range

$$
f_{i}\left(v, x_{i}\right) \approx \sum_{p, q=-K}^{K} \alpha_{i, p q} \cdot e^{\zeta_{p} v+\eta_{i, q} x_{i}}
$$

where $\zeta_{p}=j p \pi / l$ and $\eta_{i, q}=j q \pi / h_{i}$, with $l=3 \sigma_{V}$, and $h_{i}=3 \sigma_{X i} . \alpha_{i, p q}$ are the Fourier coefficients and they can be computed as

$$
\alpha_{i, p q}=\frac{1}{4 l h_{i}} \int_{\mu_{V}-l}^{\mu_{V}+l} \int_{-h}^{h} e^{-\zeta_{p} v_{1}-\eta_{i, q} v_{2}} \cdot f_{i}\left(v, x_{i}\right) d v d x_{i} .
$$

Considering that both $V$ and $X_{i}$ are within the $3 \sigma$ range, i.e., $f_{i}\left(v, x_{i}\right) \approx 0$ when $v \notin\left(\mu_{V}-l, \mu_{V}+l\right)$ or $x_{i} \notin\left(\mu_{X i}-\right.$ $\left.h_{i}, \mu_{X i}+h_{i}\right)$, (43) can be further simplified as

$$
\begin{aligned}
\alpha_{i, p q} & =\frac{1}{4 l h_{i}} E\left[e^{-\zeta_{p} V-\eta_{i, q} X_{i}}\right] \\
& =\frac{1}{4 l h_{i}} e^{-Y_{c, p}} E\left[e^{-Y_{r 1, p}-Y_{r 2, p}-\widehat{Y}_{i, p q}-\sum_{j \neq i} Y_{j, p}}\right]
\end{aligned}
$$

where

$$
\begin{aligned}
Y_{c, p} & =\zeta_{p} \cdot d_{0} \\
Y_{r 1, p} & =\zeta_{p} \cdot r_{1} R_{1} \\
Y_{r 2, p} & =\zeta_{p} \cdot r_{2} R_{2} \\
Y_{i, p} & =\zeta_{p}\left(a_{i} X_{i}+b_{i} X_{i}^{2}\right) \\
\widehat{Y}_{i, p q} & =\left(\zeta_{p} a_{i}+\eta_{i, q}\right) X_{i}+\zeta_{p} b_{i} X_{i}^{2} .
\end{aligned}
$$

Because all $X_{i}$ 's are independent, so are all $Y_{j, p}$ 's, $Y_{r 1, p}$, $Y_{r 2, p}$, and $\widehat{Y}_{i, p q}$. Then $\alpha_{i, p q}$ can be further simplified as

$$
\begin{aligned}
\alpha_{i, p q}= & \frac{1}{4 l h_{i}} e^{-Y_{c, p}} \cdot E\left[e^{-Y_{r 1, p}}\right] \cdot E\left[e^{-Y_{r 2, p}}\right] \\
& \cdot E\left[e^{-\widehat{Y}_{i, p q}}\right] \cdot \prod_{k \neq i} E\left[e^{-Y_{k, p}}\right] .
\end{aligned}
$$

As $\widehat{Y}_{i, p q}, Y_{r 1, p}, Y_{r 2, p}$, and $Y_{k, p}$ 's can be written as a general form as

$$
Y=s_{1} X_{i}+s_{2} X_{i}^{2}
$$


TABLE I

EXPERIMENT SETTING TO VERIFY $\max \left(V, X_{i}\right)$

\begin{tabular}{|c|c|c|c|c|}
\hline & $d_{0}$ & $a_{i}$ & $b_{i}$ & $r$ \\
\hline$V$ & 0 & $\{4,3,4,4\}$ & $\{1,2,1,1\}$ & 1 \\
\hline
\end{tabular}

with $s_{1}$ and $s_{2}$ being two constant values, in the following, we discuss how to compute $E\left[e^{-Y}\right]$ in its general form. By definition

$$
E\left[e^{-Y}\right]=\int_{-h_{i}}^{h_{i}} e^{-s_{1} x_{i}-s_{2} x_{i}^{2}} g_{i}\left(x_{i}\right) d x_{i}
$$

where $g_{i}\left(x_{i}\right)$ is PDF of $X_{i}$, whose range is given by $-h_{i} \leq$ $X_{i} \leq h_{i}$. For arbitrary $g_{i}\left(x_{i}\right)$, we can build a 2-D table indexed by $s_{1}$ and $s_{2}$ to compute (51).

To validate our computation of JPDF of $V$ and $X_{i}$, we compare our computed JPDF with Monte Carlo simulated JPDF. One of the examples is shown in Table I with four sources of random variables (i.e., $X_{i}$ for $i=1,2,3,4$ ) that all follow a uniform distribution. In the experiment, we compute the JPDF of $V$ and $X_{1}$. The order of Fourier series to approximate JPDF is four $(K=4)$.

With the Fourier Series approximation discussed above, we can compute the first two joint moments between $V$ and $X_{i}$. By definition, the joint moments can be computed as

$$
\begin{aligned}
E c_{i, 1} & =E\left[X_{i} \cdot \max (V, 0)\right] \\
& =\int_{-h_{i}}^{h_{i}} \int_{0}^{l+\mu_{V}} v x_{i} \cdot f_{i}\left(v, x_{i}\right) d v d x_{i} \\
E c_{i, 2} & =E\left[X_{i}^{2} \cdot \max (V, 0)\right] \\
& =\int_{-h_{i}}^{h_{i}} \int_{0}^{l+\mu_{V}} v x_{i}^{2} \cdot f_{i}\left(v, x_{i}\right) d v d x_{i} .
\end{aligned}
$$

Replacing the joint PDF $f_{i}\left(v, x_{i}\right)$ with its Fourier Series approximation, we have

$$
\begin{aligned}
E c_{i, 1} & =\int_{-h_{i}}^{h_{i}} \int_{0}^{l+\mu_{V}} v x_{i} \cdot \sum_{p, q=-K}^{K} \alpha_{i, p q} \cdot e^{\zeta_{p} v+\eta_{i, q} x_{i}} d v d x_{i} \\
& =\sum_{p, q=-K}^{K} \alpha_{i, p q} \cdot U_{p} \cdot T_{i q, 1} \\
E c_{i, 2} & =\int_{-h_{i}}^{h_{i}} \int_{0}^{l+\mu_{V}} v x_{i}^{2} \cdot \sum_{p, q=-K}^{K} \alpha_{i, p q} \cdot e^{\zeta_{p} v+\eta_{i, q} x_{i}} d v d x_{i} \\
& =\sum_{p, q=-K}^{K} \alpha_{i, p q} \cdot U_{p} \cdot T_{i q, 2}
\end{aligned}
$$

where $U_{p}, T_{i q, 1}$, and $T_{i q, 2}$ can be computed as

$$
\begin{aligned}
U_{p} & =\int_{0}^{l+\mu_{V}} v e^{\zeta_{p} v} d v \\
& = \begin{cases}\frac{1}{\eta_{p}^{2}}\left((-1)^{p} e^{\zeta_{p} \mu_{V}}\left(\zeta_{p} \mu_{V}+\zeta_{p} l-1\right)+1\right) & p \neq 0 \\
\frac{\left(l+\mu_{V}\right)^{2}}{2} & p=0\end{cases}
\end{aligned}
$$

$$
\begin{aligned}
& T_{i q, 1}=\int_{-h_{i}}^{h_{i}} x_{i} e^{\eta_{i q} x_{i}} d x_{i}= \begin{cases}(-1)^{q} \frac{2 h_{i}^{2}}{j n \pi} & q \neq 0 \\
0 & q=0\end{cases} \\
& T_{i q, 2}=\int_{-h_{i}}^{h_{i}} x_{i}^{2} e^{\eta_{i q} x_{i}} d x_{i}= \begin{cases}(-1)^{q} \frac{4 h_{i}^{3}}{n^{2} \pi^{2}} & q \neq 0 \\
\frac{2}{3} h_{i}^{3} & q=0 .\end{cases}
\end{aligned}
$$

From the above discussion, we see that the joint moments between $V$ and $X_{i}$ 's can be computed by close form formulas. Moreover, the joint moments between $\max (V, 0)$ and random variation sources, $E\left[R_{1} \max (V, 0)\right], E\left[R_{2} \max (V, 0)\right]$ can be computed in the same way.

\section{Reconstruction of the Canonical Form}

With mean of $\max (V, 0)$ and the joint moments between $\max (V, 0)$ and $X_{i}$ 's computed in the previous section, it is easy to compute the mean of $D_{m}$ and the joint moments between $D_{m}$ and $X_{i}$ 's

$$
\begin{aligned}
E\left[D_{m}\right] & =E[\max (V, 0)]+E\left[D_{2}\right] \\
= & \mu_{V m}+d_{02}+\sum b_{2 i} \\
E\left[X_{i} D_{m}\right]= & E\left[X_{i}\left(\max (V, 0)+D_{2}\right)\right] \\
= & E\left[X_{i} \max (V, 0)\right]+E\left[X_{i} D_{2}\right] \\
= & E c_{i, 1}+a_{2 i}+b_{2 i} \cdot m_{i}(3) \\
E\left[X_{i}^{2} D_{m}\right]= & E\left[X_{i}^{2} \max (V, 0)\right]+E\left[X_{i}^{2} D_{2}\right] \\
= & E c_{i, 2}+a_{2 i} \cdot m_{i}(3)+b_{2 i} \cdot m_{i}(4) \\
& +\sum_{k \neq i} b_{2 k}+d_{02} \\
E\left[R_{1} D_{m}\right]= & E\left[X_{i} \max (V, 0)\right]+E\left[R_{1} D_{2}\right] \\
= & E\left[R_{1} \max (V, 0)\right] \\
E\left[R_{2} D_{m}\right]= & E\left[X_{i} \max (V, 0)\right]+E\left[R_{2} D_{2}\right] \\
= & E\left[R_{2} \max (V, 0)\right]+r_{2} .
\end{aligned}
$$

Because we want to reconstruct $D_{m}$ in the second-order canonical form, as shown in (8), by applying the moment matching technique similar to [14], we have

$$
\begin{aligned}
E\left[D_{m}\right]= & \sum_{i=1}^{n} b_{m i}+d_{m 0} \\
E\left[X_{i} D_{m}\right]= & a_{m i}+b_{m i} \cdot m_{i}(3) \\
E\left[X_{i}^{2} D_{m}\right]= & a_{m i} \cdot m_{i}(3)+b_{m i} \cdot m_{i}(4) \\
& +\left(\sum_{k \neq i} b_{m k}+d_{m 0}\right) \\
= & a_{m i} \cdot m_{i}(3)+b_{m i} \cdot\left(m_{i}(4)-1\right) \\
& +E\left[D_{m}\right] .
\end{aligned}
$$

With the joint moments computed in (59)-(61), the constant term $d_{m 0}$ and the sensitivity coefficients $a_{m i}$ 's and $b_{m i}$ 's can be obtained by solving the linear equations above

$$
\begin{aligned}
b_{m i} & =\frac{E\left[X_{i}^{2} D_{m}\right]-E\left[D_{m}\right]-E\left[X_{i} D_{m}\right] m_{i}(3)}{m_{i}(4)-m_{i}^{2}(3)-1} \\
a_{m i} & =E\left[X_{i} D_{m}\right]-b_{m i} \cdot m_{i}(3) \\
d_{m 0} & =E\left[D_{m}\right]-\sum_{i=1}^{n} b_{m i} .
\end{aligned}
$$


Because the random term in $D_{m}$ comes from the random terms in $D_{1}$ and $D_{2}$, we assume that $r_{m} R_{m}=r_{m 1} R_{1}+r_{m 2} R_{2}$. Because the random variation sources $R_{m}, R_{1}$, and $R_{2}$ are Gaussian random variables, by applying the moment matching technique similar to (67), we have

$$
\begin{aligned}
r_{m 1} & =E\left[R_{1} \cdot D_{m}\right] \\
r_{m 2} & =E\left[R_{2} \cdot D_{m}\right] \\
r_{m} & =\sqrt{r_{m 1}^{2}+r_{m 2}^{2}}
\end{aligned}
$$

where $E\left[R_{1} \cdot D_{m}\right]$ and $E\left[R_{2} \cdot D_{m}\right]$ are computed in (63) and (64), respectively.

\section{E. Computational Complexity Analysis}

For each max operation, we need to compute $n$ joint moments between $X_{i}$ 's and $D_{m}$, where $n$ is the total number of variation sources. For each variation source, we need to compute $4(K+1)^{2}$ Fourier coefficients for the joint PDF approximation, where $K$ is the maximum order of Fourier Series. Therefore, the total computational complexity of one step max operation is $\mathcal{O}\left\{n K^{2}\right\}$. Usually, larger $K$ provides more accurate approximation. Our experiment shows that $K=4$ provides very accurate approximation for the joint PDF. For the add operation, it is easy to see that the computational complexity is $\mathcal{O}\{n\}$. The total number of max and add operations for block-based SSTA is linear to the circuit size.

Moreover, in our original algorithm for $n^{2}$ SSTA [25], the computational complexity for one step max operation is $\mathcal{O}\left\{n K^{3}\right\}$. In this paper, we have improved the algorithm and reduced the complexity to $\mathcal{O}\left\{n K^{2}\right\}$. The experimental results in Section $\mathrm{V}$ show that the improved $n^{2} S S T A$ is much faster than our original algorithm in [25]. In addition, because that our method assumes arbitrary distribution for variation sources, the distribution of variation sources does not affect the efficiency of our method, no matter the variation sources are with Gaussian distribution or not.

\section{Max Operation for Linear Delay Model}

In the previous sections, we introduce the SSTA for nonlinear delay model. In practice, when the variation scale is small, the circuit delay can be approximated as a linear function of variation sources

$$
D=d_{0}+\sum a_{i} X_{i}+r \cdot R
$$

where $X, d_{0}, A, r$, and $R$ are defined in the similar way as the second-order delay model in (4). The difference is that there are no second-order terms. Hence the atomic operations of the linear delay model are simpler than those of quadratic delay model.

For the linear delay model, we can compute the mean of $D_{m}$ in the similar way as in (40) and (59). For the joint moments, we can also apply the Fourier Series approximation method as shown in Section III-C. The only difference is that when computing the Fourier coefficients, $Y \mathrm{~s}$ in (50) is in the form of $Y=c X$. Hence $E\left[e^{Y}\right]=\mathcal{F}_{i}(-c / j)$, where $\mathcal{F}_{i}(\cdot)$ is the Fourier transformation of $g_{i}\left(x_{i}\right) . \mathcal{F}_{i}(\cdot)$ can be precomputed and stored in a 1-D lookup table. Such computation is simpler than the second order delay model. After computing the joint moments,

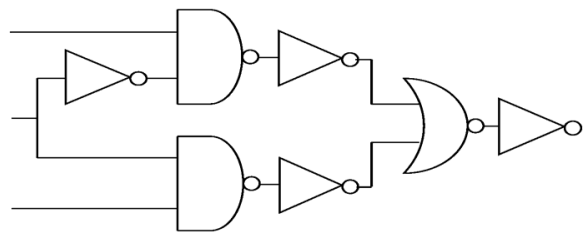

(a)

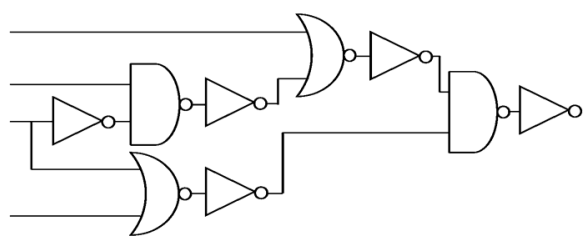

(b)

Fig. 1. Test circuits. (a) Circuit 1. (b) Circuit 2 .

we can reconstruct the linear form of $D_{m}$ in the same way as the second-order delay model as shown in Section III-D. Moreover, it is easy to find that the computational complexity of the SSTA for linear delay model is the same as that for second-order delay model.

\section{EXPERIMENTAL RESULT}

We have implemented our SSTA algorithm in C for both second-order delay model $\left(n^{2} \mathrm{SSTA}\right)$ and linear delay model (Lin-SSTA). In our experiment, we assume that the maximum order of Fourier Series approximation $K=4$ for both delay models. For comparison, we also define two comparison cases: 1) our implementation of the linear SSTA for Gaussian variation sources in [12], which we refer to as Lin-Gau and 2) 100 000sample Monte-Carlo simulation $(M C)$. We apply all the above methods to the ISCAS89 suite of benchmarks in TSMC 65-nm technology.

In our experiment, we consider two types of variation sources $L_{\mathrm{eff}}$ and $V_{\mathrm{th}}$. For each type of variation sources, inter-die, intra-die spatial, and intra-die random variations are considered. We modeled the spatial variation using the grid-based model in [26]. The number of grids (the number of spatial variation sources) is determined by the circuit size, larger circuit will have more variation sources. We also assume that the $3 \sigma$ value of the inter-die, intra-die spatial, and intra-die random variation are $10 \%, 10 \%$, and $5 \%$ of the nominal value, respectively. In the following, We perform the experiments for two variation setting: 1) both $L_{\mathrm{eff}}$ and $V_{\mathrm{th}}$ are with skew-normal distribution [27] and 2) $L_{\text {eff }}$ is with normal distribution and $V_{\text {th }}$ is with Poisson distribution. The experimental setting is shown in Table II.

In Section II, we have verified the accuracy of quadratic fitting of gate delay. In this section, we further verify the accuracy of Monte Carlo simulation with quadratic delay fitting. Because it is very time consuming to perform Monte Carlo SPICE simulation on big benchmarks, we construct two small test circuits as shown in Fig. 1. Table V compares the mean, standard deviation, and skewness for the 10000 -sample SPICE Monte Carlo simulation, 10 000-Monte Carlo simulation with quadratic delay fitting, and $n^{2}$ SSTA under variation setting (1). From the table, we see that the result of Monte Carlo simulation with quadratic 
TABLE II

Experiment Setting. The $3 \sigma$ Value is the Normalized With Respect to the Nominal Value

\begin{tabular}{|c|c|c|c|c|c|}
\hline & $L_{\text {eff }}$ distribution & $V_{t h}$ distribution & $3 \sigma$ inter-die & $3 \sigma$ spatial & $3 \sigma$ random \\
\hline Case $(1)$ & Skewnormal & Skewnormal & $10 \%$ & $10 \%$ & $5 \%$ \\
\hline Case $(2)$ & Normal & Poisson & $10 \%$ & $10 \%$ & $5 \%$ \\
\hline
\end{tabular}

TABLE III

Comparison Between SPiCE Simulation, Monte Carlo Simulation and $n^{2}$ SSTA

\begin{tabular}{|c||c|c|c||c|c|c||c|c|c|}
\hline \multirow{2}{*}{$\begin{array}{c}\text { bench } \\
\text { mark }\end{array}$} & \multicolumn{3}{c||}{ SPICE MC } & \multicolumn{3}{c||}{ MC with quad fitting } & \multicolumn{3}{c|}{$n^{2} S S T A$} \\
\cline { 2 - 10 } & $\mu(\mathrm{ps})$ & $\sigma(\mathrm{ps})$ & $\gamma$ & $\mu(\mathrm{ps})$ & $\sigma(\mathrm{ps})$ & $\gamma$ & $\mu(\mathrm{ps})$ & $\sigma(\mathrm{ps})$ & $\gamma$ \\
\hline circuit1 & 541.2 & 98.3 & 0.52 & $543.4(+0.41 \%)$ & $98.9(+0.61 \%)$ & $0.54(+3.8 \%)$ & $544.2(+0.55 \%)$ & $99.1(+0.8 \%)$ & $0.55(+5.8 \%)$ \\
circuit2 & 792.2 & 124.3 & 0.59 & $795.5(+0.4 \%)$ & $126.2(+1.5 \%)$ & $0.61(+3.3 \%)$ & $796.1(+0.5 \%)$ & $127.1(+2.3 \%)$ & $0.64(+7.9 \%)$ \\
\hline
\end{tabular}

TABLE IV

Error Percentage of Mean, Standard Deviation, Skewness, and 95\% Percentile Point for Variation Setting (1). Note: the Error Percentage of Mean, Standard Deviation, and 95\% Percentile Point is Computed as $100 \times($ MC_value - SSTA_value $) / \sigma_{M C}$, AND the ERror Percentage of Skewness is Computed as $100 \times\left(\gamma_{M C}-\gamma_{\mathrm{SSTA}}\right) / \gamma_{M C}$

\begin{tabular}{|c|c|c|c|c|c|c|c|c|c|c|c|c|c|c|c|c|c|c|c|}
\hline \multirow{2}{*}{$\begin{array}{l}\text { bench } \\
\text { name }\end{array}$} & \multirow[t]{2}{*}{ G } & \multirow[t]{2}{*}{$\mathrm{N}$} & \multicolumn{5}{|c|}{$n^{2}$ SSTA } & \multicolumn{5}{|c|}{ Lin-SSTA } & \multicolumn{5}{|c|}{ Lin-Gau } & [25] & $\overline{\mathrm{MC}}$ \\
\hline & & & $\mu$ & $\bar{\sigma}$ & $\gamma$ & $95 \%$ & $\mathrm{~T}$ & $\bar{\mu}$ & $\bar{\sigma}$ & $\bar{\gamma}$ & $95 \%$ & $\mathrm{~T}$ & $\mu$ & $\bar{\sigma}$ & $\gamma$ & $95 \%$ & $\mathrm{~T}$ & $\mathrm{~T}$ & $\mathrm{~T}$ \\
\hline s27 & 8 & 8 & 0.25 & -0.71 & 7.34 & 1.23 & 0.65 & 0.41 & 3.45 & -27.23 & -4.67 & 0.32 & -1.23 & -4.06 & -100 & -23.14 & 0.01 & 1.12 & 1.71 \\
\hline s344 & 101 & 18 & 0.63 & -1.18 & -2.24 & 1.21 & 1.13 & 1.02 & -1.66 & -17.35 & -3.89 & 0.61 & -1.10 & 1.52 & -100 & -20.82 & 0.02 & 5.54 & 34.29 \\
\hline s386 & 118 & 18 & 0.67 & -1.40 & -3.00 & 1.04 & 1.18 & 0.53 & -2.41 & -15.94 & 6.50 & 0.65 & 1.07 & -1.43 & -100 & -21.82 & 0.02 & 6.49 & 36.03 \\
\hline$s 420$ & 140 & 25 & 0.69 & -1.25 & -3.21 & 1.43 & 1.36 & -0.75 & 1.71 & -18.79 & -4.06 & 0.69 & 1.33 & 1.24 & -100 & -25.30 & 0.02 & 7.35 & 39.06 \\
\hline s444 & 119 & 25 & -0.61 & -1.06 & -4.34 & -1.16 & 1.38 & -1.31 & 2.06 & -24.07 & -6.18 & 0.67 & -1.33 & 1.28 & -100 & -29.26 & 0.02 & 8.69 & 41.10 \\
\hline s832 & 262 & 33 & -0.31 & -1.08 & 6.91 & -1.47 & 1.92 & 0.28 & -4.99 & -27.40 & -2.82 & 0.85 & 1.14 & -4.71 & -100 & -16.47 & 0.04 & 10.25 & 102.7 \\
\hline s953 & 311 & 43 & 0.78 & -1.15 & -2.36 & 1.63 & 1.96 & -0.84 & 2.09 & -17.18 & -5.65 & 0.87 & 1.29 & 1.18 & -100 & -25.36 & 0.05 & 11.32 & 155.7 \\
\hline s1196 & 388 & 55 & -0.30 & -0.69 & 8.17 & -1.04 & 2.17 & -0.30 & -4.89 & -35.69 & 2.20 & 0.96 & 1.31 & -4.40 & -100 & -21.79 & 0.05 & 13.13 & 172.2 \\
\hline s1494 & 588 & 68 & -0.78 & -1.30 & -3.69 & -1.77 & 2.27 & 1.20 & 1.75 & -16.15 & 5.88 & 1.03 & -1.05 & 1.16 & -100 & -28.69 & 0.06 & 15.52 & 323.4 \\
\hline s5378 & 1004 & 82 & -0.65 & -1.13 & -2.67 & -1.65 & 5.37 & 0.73 & 2.22 & -22.54 & 6.14 & 2.26 & -1.00 & 1.49 & -100 & -30.09 & 0.07 & 27.32 & 1256 \\
\hline s9234 & 2027 & 99 & -1.18 & -0.18 & 2.85 & -0.64 & 6.83 & 0.65 & -1.69 & -17.37 & 4.15 & 3.14 & -3.07 & -1.47 & -100 & -31.81 & 0.09 & 32.18 & 2769 \\
\hline s13207 & 2573 & 115 & -0.93 & -0.25 & 2.30 & -0.52 & 9.02 & 0.60 & 1.75 & -21.27 & 5.74 & 4.22 & 2.29 & -1.86 & -100 & -26.34 & 0.11 & 47.35 & 4715 \\
\hline s15850 & 3448 & 135 & -1.51 & -0.21 & 2.87 & -0.51 & 11.36 & 0.70 & 2.32 & -20.45 & 3.44 & 5.28 & 3.35 & 1.33 & -100 & -33.16 & 0.15 & 58.25 & 6891 \\
\hline s38417 & 8709 & 176 & -0.25 & -0.91 & 6.14 & -1.03 & 23.37 & -2.14 & 3.53 & -30.24 & -3.38 & 10.00 & -1.11 & -5.30 & -100 & -16.58 & 0.28 & 98.13 & 19061 \\
\hline s38584 & 11448 & 176 & -1.41 & -0.27 & 2.42 & -0.55 & 25.39 & -1.95 & -0.90 & -19.85 & 3.96 & 13.49 & -2.40 & -1.82 & -100 & -28.40 & 0.31 & 104.1 & 19203 \\
\hline Ave & - & - & 0.73 & 0.85 & 4.03 & 1.13 & - & 0.89 & 2.49 & 22.15 & 4.58 & 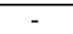 & 1.60 & 2.58 & 100 & 25.30 & - & - & - \\
\hline
\end{tabular}

TABLE V

Error Percentage of Mean, Standard Deviation, Skewness, and 95\% Percentile Point for Variation Setting (2)

\begin{tabular}{|c|c|c|c|c|c|c|c|c|c|c|c|c|c|c|c|c|c|c|c|}
\hline \multirow{2}{*}{$\begin{array}{l}\text { bench } \\
\text { name }\end{array}$} & \multirow[t]{2}{*}{ G } & \multirow[t]{2}{*}{$\mathrm{N}$} & \multicolumn{5}{|c|}{$n^{2}$ SSTA } & \multicolumn{5}{|c|}{ Lin-SSTA } & \multicolumn{5}{|c|}{ Lin-Gau } & {$[25]$} & $\mathrm{MC}$ \\
\hline & & & $\mu$ & $\sigma$ & $\bar{\gamma}$ & $95 \%$ & $\mathrm{~T}$ & $\mu$ & $\sigma$ & $\bar{\gamma}$ & $95 \%$ & $\mathrm{~T}$ & $\mu$ & $\sigma$ & $\bar{\gamma}$ & $95 \%$ & $\mathrm{~T}$ & $\mathrm{~T}$ & $T$ \\
\hline s27 & 8 & 8 & 0.29 & -0.93 & 8.21 & 1.07 & 0.64 & 0.37 & -3.39 & -30.01 & -4.06 & 0.32 & -1.24 & -4.15 & -100 & $\mid-22.71$ & 0.01 & 1.11 & 1.74 \\
\hline s344 & 101 & 18 & -0.66 & -1.40 & -2.29 & -1.26 & 1.12 & -1.20 & -1.91 & -19.22 & 4.17 & 0.61 & -1.12 & 1.55 & -100 & -21.58 & 0.02 & 5.34 & 33.84 \\
\hline s386 & 118 & 18 & -0.65 & -1.36 & -2.93 & -1.20 & 1.17 & 0.60 & 1.71 & -19.13 & 6.65 & 0.64 & 1.08 & -1.44 & -100 & -22.72 & 0.02 & 6.52 & 35.92 \\
\hline$s 420$ & 140 & 25 & -0.61 & -1.28 & -4.50 & -1.45 & 1.38 & -0.63 & -1.71 & -20.04 & -5.44 & 0.66 & 1.30 & 1.19 & -100 & -24.26 & 0.02 & 7.33 & 38.45 \\
\hline s444 & 119 & 25 & -0.87 & -1.52 & -3.20 & -1.19 & 1.38 & -1.26 & 2.12 & -23.03 & -7.41 & 0.69 & -1.43 & 1.30 & -100 & -29.81 & 0.02 & 8.74 & 40.36 \\
\hline s832 & 262 & 33 & 0.27 & -0.90 & 8.75 & 1.30 & 1.87 & -0.26 & 4.86 & -30.64 & 3.24 & 0.84 & 1.13 & -4.63 & -100 & -16.88 & 0.04 & 10.39 & 99.4 \\
\hline s953 & 311 & 43 & 0.79 & -1.16 & -3.30 & 1.34 & 1.92 & -0.68 & -1.68 & -14.91 & -5.19 & 0.90 & 1.25 & 1.09 & -100 & -25.09 & 0.05 & 11.46 & 152.6 \\
\hline s1196 & 388 & 55 & 0.33 & -0.69 & 6.32 & 1.38 & 2.13 & -0.24 & 4.59 & -31.14 & 2.75 & 0.93 & 1.39 & -4.02 & -100 & -21.74 & 0.05 & 13.17 & 173.9 \\
\hline s1494 & 588 & 68 & -0.85 & -1.55 & -3.46 & -1.82 & 2.37 & 1.48 & -2.54 & -14.35 & 7.10 & 1.03 & -1.03 & 1.21 & -100 & -26.44 & 0.06 & 15.78 & 317.6 \\
\hline s5378 & 1004 & 82 & 0.71 & -1.20 & -3.61 & 1.76 & 5.38 & -0.74 & 2.38 & -20.69 & -5.59 & 2.34 & -1.02 & 1.45 & -100 & -28.92 & 0.07 & 27.12 & 1232 \\
\hline s9234 & 2027 & 99 & 1.05 & -0.23 & 2.22 & 0.53 & 6.77 & 0.61 & -1.64 & -16.17 & 4.52 & 3.15 & -3.24 & -1.39 & -100 & -31.05 & 0.09 & 32.60 & 2804 \\
\hline s13207 & 2573 & 115 & 1.16 & -0.25 & 2.49 & 0.72 & 8.83 & -0.56 & -1.83 & -18.04 & -5.53 & 4.17 & 2.40 & -1.84 & -100 & -26.56 & 0.11 & 48.05 & 4639 \\
\hline s15850 & 3448 & 135 & 1.22 & -0.24 & 2.95 & 0.51 & 11.40 & 0.79 & -1.72 & -19.77 & 3.64 & 5.16 & 3.34 & 1.35 & -100 & -33.65 & 0.15 & 57.15 & 6653 \\
\hline s38417 & 8709 & 176 & 0.25 & -0.70 & 6.17 & 1.20 & 23.23 & 1.76 & 3.54 & -27.25 & 3.47 & 10.28 & -1.15 & -5.11 & -100 & -16.60 & 0.28 & 99.22 & 19470 \\
\hline s38584 & 11448 & 176 & -1.56 & -0.21 & 1.92 & -0.54 & 25.28 & 2.17 & 0.97 & -21.92 & -4.13 & 13.39 & -2.59 & -1.72 & -100 & -29.39 & 0.31 & 105.0 & 19891 \\
\hline Ave & - & - & 0.75 & 0.91 & 4.16 & 1.15 & - & 0.89 & 2.44 & 21.8 & 4.86 & 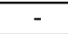 & 1.65 & \begin{tabular}{|l|}
2.53 \\
\end{tabular} & 100 & 25.2 & - & - & - \\
\hline
\end{tabular}

delay fitting is very close to the SPICE Monte Carlo simulation. In the rest of this section, we will use the Monte Carlo simulation with quadratic delay fitting as the golden case for comparison.

Fig. 2 illustrates the PDF comparison for circuit s15850 under variation setting (1). From the figure, we find that compared to the Monte-Carlo simulation, $n^{2}$ SSTA is the most accurate, the next is Lin-SSTA. Both of our SSTA methods are more accurate than Lin-Gau. Such result is reasonable because $n^{2} \mathrm{SSTA}$ captures the both nonlinear and linear effects while Lin-SSTA captures only linear effect. Lin-Gau is for Gaussian variation sources only, and therefore it does not work well under nonGaussian variation sources.

Table V compares the run time in second $(T)$, and the error percentage of mean $(\mu)$, standard deviation $(\sigma)$, skewness $(\gamma)$, and $95 \%$ percentile point $(95 \%)$ under variation setting (1). In the table, the error percentage of mean, standard deviation and $95 \%$ percentile point is computed as $100 \times\left(M C_{-}\right.$Value SSTA_value) $/ \sigma_{M C}$, and the error percentage of skewness is computed as $100 \times\left(\gamma_{M C}-\gamma_{\mathrm{SSTA}}\right) / \gamma_{M C}$. Moreover, the average error in the table is average of the absolute value. From 


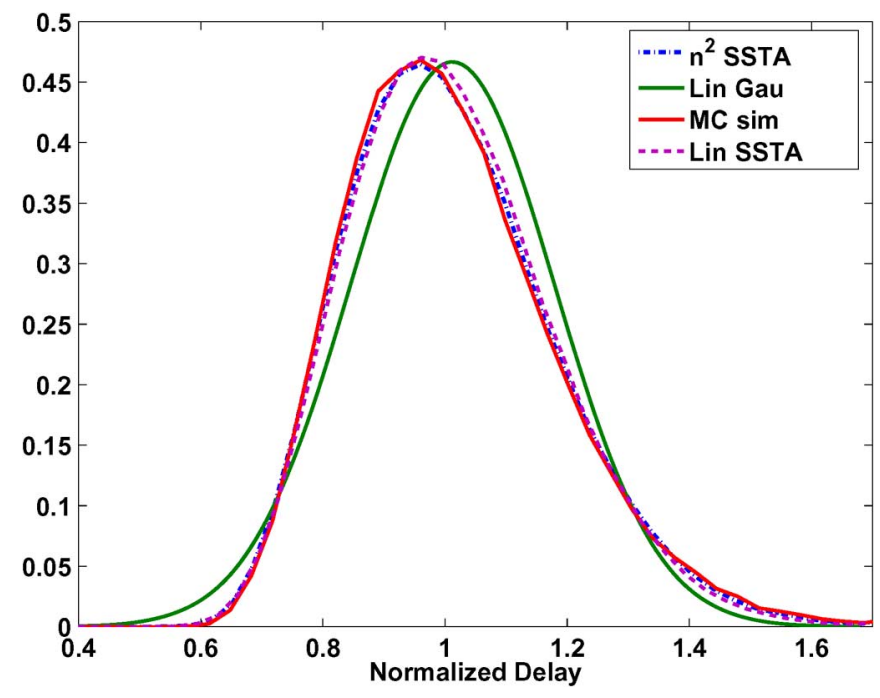

Fig. 2. PDF comparison for circuit s15850.

the table, we see that for $n^{2}$ SSTA the error of mean, standard deviation and $95 \%$ percentile point is within $2 \%$, and the error of skewness is within $8 \%$. The Lin-SSTA results similar mean deviation error. Compared to $n^{2}$ SSTA, the error of standard deviation and $95 \%$ percentile point is a little bit higher, but the error of skewness is much larger. This is because Lin-SSTA ignores all nonlinear effects which significantly affect the skewness. The error of Lin-Gau is larger than both of our SSTA methods, especially for skewness. This is because Lin-Gau is for Gaussian variation sources and cannot capture the skewness of the non-Gaussian variation sources. Moreover, we also find that the run time of all SSTA methods are linear to the circuit size. The run time of our methods is larger than that of Lin-Gau, but it is still acceptable and is significantly shorter than that of the Monte-Carlo simulation. In the table, we also compare the run time of our algorithm in this paper to that of our original method [25]. We see the approach in this paper is about $5 \times$ faster than our original method. Because the basic algorithms of this paper and our original method in [25] are the same, the approach in this paper provides the same accuracy as our original method.

Table V illustrates the results under variation setting (2). It can be found that our methods are still very accurate under such variation setting. For the $n^{2} \mathrm{SSTA}$, the error of mean, standard deviation, and $95 \%$ percentile point is within $2 \%$, and the error of skewness is within $10 \%$. This shows that our approach works well for different distributions.

\section{CONCLUSION}

A novel method to handle the max operation has been presented to handle both quadratic delay dependency and non-Gaussian variation sources simultaneously. An SSTA flow, $n^{2}$ SSTA, has been developed based on such max operation. We have shown that all statistical atomic operations can be performed efficiently via either closed-form formulas or low dimension (at most 2-D) lookup table. It has been proved that the complexity of $n^{2} \mathrm{SSTA}$ is linear in both variation sources and circuit sizes. Compared to Monte Carlo simulation for non-Gaussian variations and nonlinear delay models, our approach predicts the mean, standard deviation and $95 \%$ percentile point with less than $2 \%$ error, and the skewness with less than $10 \%$ error.

In the future, we will extend our work to consider more general delay models, such as non-polynomial delays and/or dependency on variations' cross terms. Moreover, in this paper, we use deterministic slew rate model. We will also try to consider statistical slew model in our future research.

\section{REFERENCES}

[1] S. Nassif, "Modeling and analysis of manufacturing variations," in Proc. IEEE Int. Conf. Custom Integr. Circuits, 2001, pp. 223-228.

[2] F. Gong, H. Yu, and L. He, "PiCAP: A parallel and incremental fullchip capacitance extraction considering random process variation," in Proc. IEEE/ACM Design Autom. Conf., 2009, pp. 764-769.

[3] C. Murthy and M. Gall, "Process variation effects on circuit performance: TCAD simulation of 256-Mbit technology DRAMs," IEEE Trans. Comput.-Aided Design Integr. Circuits Syst., vol. 16, no. 11, pp. 1383-1389, Nov. 1997.

[4] F. Gong, H. Yu, Y. Shi, D. Kim, J. Ren, and L. He, "Quickyield: An efficient global-search based parametric yield estimation with performance constraints," in Proc. IEEE/ACM Design Autom. Conf., 2010, pp. 392-397.

[5] L. T. Pillage and R. A. Rohrer, "Asymptotic waveform evaluation for timing analysis," IEEE Trans. Comput.-Aided Design Integr. Circuits Syst., vol. 9, no. 4, pp. 352-366, Apr. 1990.

[6] F. Gong, H. Yu, and L. He, "Stochastic analog circuit behavior modeling by point estimation method," in Proc. Int. Symp. Phys. Design, 2011, pp. 175-182.

[7] J.-J. Liou, A. Krstic, L.-C. Wang, and K.-T. Cheng, "False-path-aware statistical timing analysis and efficient path selection for delay testing and timing validation," in Proc. IEEE/ACM Design Autom. Conf., 2002, pp. 566-569.

[8] J. Jess, K. Kalafala, S. Naidu, R. Otten, and C. Visweswariah, "Statistical timing for parametric yield prediction of digital integrated circuits," in Proc. IEEE/ACM Design Autom. Conf., 2003, pp. 932-937.

[9] M. Orshansky and A. Bandyopadhyay, "Fast statistical timing analysis handling arbitrary delay correlations," in Proc. IEEE/ACM Design Autom. Conf., 2004, pp. 337-342.

[10] A. Ramalingam, A. K. Singh, S. R. Nassif, G.-J. Nam, M. Orshansky, and D. Z. Pan, "An accurate sparse matrix based framework for statistical static timing analysis," in Proc. IEEE/ACM Int. Conf. Comput.Aided Design, 2006, pp. 231-236.

[11] H. Chang and S. Sapatnekar, "Statistical timing analysis considering spatial correlations using a single PERT-like traversal," in Proc. IEEE/ACM Int. Conf. Comput.-Aided Design, 2003, pp. 621-625.

[12] C. Visweswariah, K. Ravindran, K. Kalafala, S. Walker, and S. Narayan, "First-order incremental block-based statistical timing analysis," in Proc. IEEE/ACM Design Autom. Conf., 2004, pp. 331-336.

[13] L. Zhang, W. Chen, Y. Hu, J. A. Gubner, and C. C.-P. Chen, "Correlation-preserved non-gaussian statistical timing analysis with quadratic timing model," in Proc. IEEE/ACM Design Autom. Conf., 2005, pp. 83-88.

[14] Y. Zhan, A. J. Strojwas, X. Li, and L. T. Pileggi, "Correlation-aware statistical timing analysis with non-gaussian delay distribution," in Proc. IEEE/ACM Design Autom. Conf., 2005, pp. 77-82.

[15] V. Khandelwal and A. Srivastava, "A general framework for accurate statistical timing analysis considering correlations," in Proc. IEEE/ACM Design Autom. Conf., 2005, pp. 89-94.

[16] J. Singh and S. Sapatnekar, "Statistical timing analysis with correlated non-gaussian parameters using independent componenet analysis," in Proc. ACM/IEEE Int. Workshop Timing Issues, 2006, pp. 143-148.

[17] H. Chang, V. Zolotov, C. Visweswariah, and S. Narayan, "Parameterized block-based statistical timing analysis with non-Gaussian and nonlinear parameters," in Proc. IEEE/ACM Design Autom. Conf., 2005, pp. $71-76$.

[18] S. Bhardwaj, P. Ghanta, and S. Vrudhula, "A framework for statistical timing analysis using non-linear delay and slew models," in Proc. IEEE/ACM Int. Conf. Comput.-Aided Design, 2006, pp. 225-230.

[19] Y. Zhan, A. J. Strojwas, D. Newmark, and M. Sharma, "Generic statistical timing analysis with non-gaussian process parameters," in Proc. Austin Conf. Integr. Syst. Circuits, 2006, pp. 71-76. 
[20] X. Li, J. Le, and P. Pileggi, "Asymptotic probability extraction for nonnormal distributions of circuit performance," in Proc. IEEE/ACM Int. Conf. Comput.-Aided Design, 2004, pp. 2-9.

[21] L. Zhang, W. Chen, Y. Hu, and C. C. ping Chen, "Statistical static timing analysis with conditional linear MAX/MIN approximation and extended canonical timing model," IEEE Trans. Comput.-Aided Design Integr. Circuits Syst., vol. 25, no. 6, pp. 1183-1191, Jun. 2006.

[22] L. Cheng, J. Xiong, and L. He, "Non-gaussian statistical timing analysis using second-order polynomial fitting," IEEE Trans. Comput.-Aided Design Integr. Circuits Syst., vol. 28, no. 1, pp. 130-140, Jan. 2009.

[23] L. Cheng, J. Xiong, and L. He, "Non-gaussian statistical timing analysis using Second-Order polynomial fitting," in Proc. Asia South Pacific Design Autom. Conf., 2008, pp. 298-303.

[24] L. Zhang, J. Shao, and C. C.-P. Cheng, "Non-Gaussian statistical parameter modeling for ssta with confidence interval analysis," in Proc. Int. Symp. Phys. Design, 2006, pp. 33-38.

[25] L. Cheng, J. Xiong, and L. He, "Non-linear statistical static timing analysis for non-gaussian variation sources," in Proc. ACM/IEEE Int. Workshop on Timing Issues, 2007, pp. 143-148.

[26] J. Xiong, V. Zolotov, and L. He, "Robust extraction of spatial correlation," IEEE Trans. Comput.-Aided Design Integr. Circuits Syst., vol. 26, no. 4, pp. 619-631, Apr. 2007.

[27] A. Azzalini, "The skew-normal distribution and related multivariate families," Scandinavian J. Stat., vol. 32, no. 2, pp. 159-188, Jun. 2005.

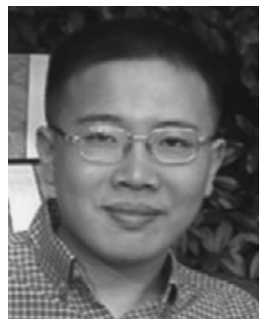

Lerong Cheng (M'10) received the B.S. degree in electronics and communication engineering from Zhongshan University, Guangzhou, China, in 2001, the M.S. degree in electrical and computer engineering from Portland State University, Portland, $\mathrm{OR}$, in 2003, and the Ph.D. degree in electrical engineering from the University of California, Los Angeles, in 2009.

He is currently a Computer-Aided Design Engineer with SanDisk Corporation, Milpitas, CA. His current research interests include computer-aided design of very large scale integration circuits and systems, programmable fabrics, low-power and high-performance designs, and statistical timing analysis.

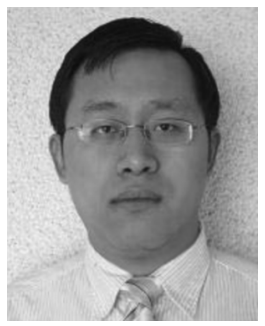

Fang Gong (S'08) received the B.S. degree from the Computer Science Department, Beijing University of Aeronautics and Astronautics, Beijing, China, in 2005, the M.S. degree from the Computer Science Department, Tsinghua University, Beijing, China, in 2008, and the Ph.D. degree from the Electrical Engineering Department, University of California, Los Angeles.

His research interests mainly focus on numerical computing and stochastic techniques for $\mathrm{CAD}$, including fast circuit simulation, yield estimation and optimization. He also works on numerics parallel and distributed computing.

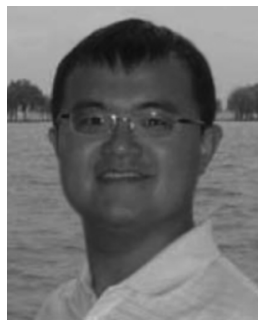

Wenyao Xu (S'08) received the B.S. and M.S degrees in electrical engineering from Zhejiang University, Hangzhou, China, in 2006 and 2008, respectively. He is currently pursuing the Ph.D. degree from the Department of Electrical Engineering and Computer Sciences, University of California at Los Angeles.

His research interests include computer-aided design for programmable fabrics, wireless health, and brain computer interface.

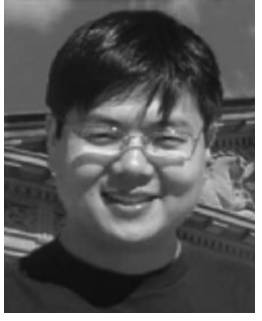

Jinjun Xiong (S'04-M'06) received the B.E. (with honors) degree in precision instrument, the B.E. degree in industrial engineering, and the M.E. degree in precision instruments from Tsinghua University, Beijing, China, in 1998, 1998, and 2000, respectively, the M.S. degree in electrical and computer engineering from the University of Wisconsin, Madison, in 2002, and the $\mathrm{Ph} . \mathrm{D}$. degree in electrical engineering from the University of California, Los Angeles (UCLA), in 2006 .

He is currently a Research Staff Member with the IBM Thomas J. Watson Research Center, Yorktown Heights, NY. His research interests include statistical timing analysis and optimization, design for manufacturability, design automation for VLSI circuits and systems, largescale optimization, and combinatorial mathematics.

Dr. Xiong was the recipient of the 2005-2006 Outstanding Ph.D. Award in Electrical Engineering from UCLA, the Best Student Paper Award at the International Conference on ASIC 2003, and the Best Paper Award at the ACM International Symposium on Physical Design in 2006.

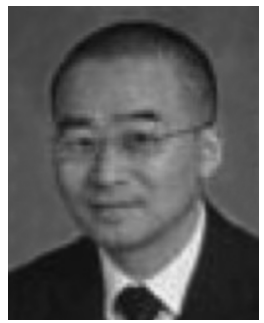

Lei He (M'99-SM'08) received the Ph.D. degree in computer science from the University of California, Los Angeles (UCLA), in 1999.

$\mathrm{He}$ is a Professor with the Electrical Engineering Department, University of California, Los Angeles (UCLA), and was a faculty member at University of Wisconsin, Madison, between 1999 and 2002. $\mathrm{He}$ also held visiting or consulting positions with Cadence, Empyrean Soft, Hewlett-Package, Intel, and Synopsys, and was technical advisory board member for Apache Design Solutions and Rio Design Automation. His research interests include modeling and simulation, VLSI circuits and systems, and cyber physical systems. He has published one book and over 200 technical papers with 12 best paper nominations mainly from Design Automation Conference and International Conference on Computer-Aided Design.

Dr. He was a recipient of Five Best Paper or Best Contribution Awards including the ACM Transactions on Electronic System Design Automation 2010 Best Paper Award.

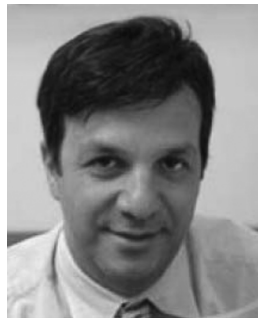

Majid Sarrafzadeh (F'96) received the Ph.D. degree in electrical and computer engineering from the University of Illinois at Urbana-Champaign, UrbanaChampaign, in 1987.

He joined Northwestern University, Evanston, IL, as an Assistant Professor in 1987. In 2000, he joined the Computer Science Department, University of California at Los Angeles (UCLA). He is currently a co-director of the UCLA Wireless Health Institute where he has a few dozen active project with medical doctors and nurses around the world. His recent research interests lie in the area of Embedded and Reconfigurable Computing with emphasis on healthcare. He has published approximately 370 papers, co-authored 5 books, and is a named inventor on many US patents. Dr. Sarrafzadeh has collaborated with many industries in the past 25 years industries and was the architect of Monterey Design Systems C Synopsys acquired the company. He was a co-founder of Hier Design, Inc. Hier Design was acquired by Xilinx in 2004. He has recently co-founded Medisens and BioAssyst: both companies in the area of Wireless Health.

Dr. Sarrafzadeh has served on the technical program committee of numerous conferences and been a general chair of many of them. 